\title{
Two-Dice Horse Race
}

\section{Colin Foster}

School of Education, University of Nottingham, Nottingham, UK

e-mail: colin.foster@ nottingham.ac.uk

\section{David Martin}

School of Education, University of Nottingham, Nottingham, UK

e-mail: d.martin@virginmedia.com

\begin{abstract}
In this article we analyse the "two-dice horse race" task often used in lower secondary school, in which two ordinary dice are thrown repeatedly and each time the sum of the scores determines which horse (numbered 1 to 12) moves forwards one space. The process is repeated until one horse crosses the finishing line a fixed number of spaces away. We examine the difficulties involved in calculating the probability of any particular horse winning the race and carry out a simulation to estimate these probabilities. We conclude that while this task might be a helpful way to introduce sample space diagrams, great care is needed in interpreting the outcome of the race. The greater probability of obtaining a total score of 7 on a single throw of two dice is only partly responsible for horse 7's greater chance of winining, since the length of the track is also important.
\end{abstract}

Keywords: Teaching statistics; Sample space diagrams; Numbers of possibilities; Probability calculations; Simulations to estimate probabilities; Two-dice experiment; Expected values; Markov processes

\section{INTRODUCTION}

One of us recently saw the classic "two-dice horse race" lesson in which the teacher asked a class of Year 8 pupils (age 12-13) to throw two ordinary dice and add the scores to determine which horse (numbered 1 to 12 ) would move forwards one space. The process was repeated until one of the horses had crossed the finishing line, which, for a track length of 10, was 10 spaces away. [Note 1] The intention was for pupils to appreciate:

(i) that horse 1 would never leave the starting gate, since there is no way to score a total of 1 with two dice;

(ii) that horses 2 to 12 are not equally likely to win, and that this must be related to unequal chances of winning at each throw of the two dice;

(iii) that a sample space diagram enables calculation of the probability of each horse moving one space, and of a particular horse winning in very simplified versions of this race. 
In the lesson, the pupils carried out the experiment with enthusiasm and soon began to realise that horse number 7 was generally the best bet. This motivated them to calculate the probabilities of each horse winning for a track of length 1 - that is, the probabilities of the various totals when throwing two fair dice - using the relevant sample space diagram (Blinded Reference). However, at this point some of the pupils seemed to assume that because the probability of getting a 7 with two dice was $\frac{1}{6}$, the probability that horse 7 would win the race was also $\frac{1}{6}$. This raised several possible questions, many of which could be tackled through simulations, and some of which can be framed in suitable ways for (possibly older) students to investigate. Intuitively the probability of horse 7 winning is greater than $\frac{1}{6}$, but how much greater, and how can this be shown? In general, what is the chance of a particular horse winning and how does this depend on the track length? To calculate these probabilities analytically is difficult. Below we consider some aspects of a two-horse race, and then a simulation of the original race.

\section{SIMPLER RACES}

Let's consider two horses, call them Ned and Pete, with the probability of Ned moving forwards one space each "throw" being $p$ and the corresponding probability for Pete being $q$ $=1-p$. For some classes you might wish to use specific values for $p$, but make $p>1 / 2$. Below are some activities that could be suitable for different classes.

(a) For a track length of just 2, Ned can win in two steps or in three. Students can use a sample space diagram to show that the chance of Ned winning is $p^{2}+2 p^{2} q=p^{2}(3-$ $2 p$ ). Younger students could do this for selected values of $p$, and older students who can factorise simple quadratics could show that this is greater than $p$ provided that $p>$ $1 / 2$.

(b) For a track length of 3, ask students how many steps it could take for Ned to win (3, 4 or 5). Depending on the class, students could again use sample space diagrams to find the chance of Ned winning, either for a specific value of $p$ or generally in terms of $p$.

(c) This could lead to asking how many steps it could take for Ned to win for longer track lengths. Again, the question can be made specific or can be generalised depending on the class.

(d) With three horses on a track length of two, students could again be asked how many steps it could take for Ned to win (2, 3 or 4 this time). Again, if desired, a sample space diagram could be used to find the chance of Ned winning.

\section{RETURNING TO THE "TWO-DICE HORSE RACE"}

With our original 11 horses (not counting horse 1), even for a track length of 2, calculating the probability that any particular horse (say, horse number 7) will win is complicated, as we 
have to consider what all the other horses might be doing. They will each move 0 or 1 spaces, and, as these other horses have different probabilities from each other of moving, this is a very messy calculation (an indication is given in Appendix 1 and a full general approach is outlined in Appendix 2).

An important question is how many steps (two-dice throws) it might take for any particular horse to win. For a track of length 2, the minimum is 2 and the maximum is 12 . The maximum occurs when all the other 10 horses move one space and then the winning horse moves two spaces. Older students could consider the general question of the number of possible steps for a horse to win when there are $h$ horses with a track length of $l$ [Note 2].

As the full horse race is clearly far too complicated for students aged 9-19 to tackle analytically, there is the opportunity to demonstrate the usefulness of simulations to estimate probabilities, with a large number of simmulations allowing very accurate estimations. We implemented a simulation [Note 3] in which we ran the horse race 1,000,000 times for different lengths of track and found by repeating each simulation a few times that the results were consistent to 3 decimal places (Table 1). We can see from Figure 1 that the distribution becomes more peaked at horse 7 as the track length increases.

\begin{tabular}{|c|c|c|c|c|c|c|c|c|c|c|}
\hline & \multicolumn{10}{|c|}{ Track Length } \\
\hline & 1 & 2 & 3 & 4 & 5 & 6 & 7 & 8 & 9 & 10 \\
\hline 2 & 0.028 & 0.009 & 0.003 & 0.001 & 0.001 & 0.000 & 0.000 & 0.000 & 0.000 & 0.000 \\
\hline 3 & 0.056 & 0.034 & 0.021 & 0.014 & 0.009 & 0.006 & 0.004 & 0.003 & 0.002 & 0.001 \\
\hline 4 & 0.083 & 0.069 & 0.057 & 0.047 & 0.039 & 0.032 & 0.027 & 0.023 & 0.019 & 0.016 \\
\hline 5 & 0.111 & 0.113 & 0.110 & 0.105 & 0.099 & 0.093 & 0.088 & 0.083 & 0.078 & 0.074 \\
\hline 6 & 0.139 & 0.164 & 0.179 & 0.187 & 0.193 & 0.197 & 0.199 & 0.201 & 0.201 & 0.202 \\
\hline 7 & 0.167 & 0.221 & 0.261 & 0.293 & 0.320 & 0.343 & 0.363 & 0.382 & 0.399 & 0.415 \\
\hline 8 & 0.139 & 0.164 & 0.179 & 0.187 & 0.193 & 0.197 & 0.199 & 0.201 & 0.201 & 0.202 \\
\hline 9 & 0.111 & 0.113 & 0.110 & 0.105 & 0.099 & 0.094 & 0.088 & 0.083 & 0.078 & 0.074 \\
\hline 10 & 0.083 & 0.069 & 0.057 & 0.047 & 0.039 & 0.032 & 0.027 & 0.023 & 0.019 & 0.016 \\
\hline 11 & 0.056 & 0.034 & 0.021 & 0.014 & 0.009 & 0.006 & 0.004 & 0.003 & 0.002 & 0.001 \\
\hline 12 & 0.028 & 0.009 & 0.003 & 0.001 & 0.001 & 0.000 & 0.000 & 0.000 & 0.000 & 0.000 \\
\hline
\end{tabular}

Table 1. The probability (correct to 3 decimal places) of each horse winning races of different track lengths.

For students who have met expected values, there is more of interest. For example, consider horses 6 and 7 . We know that $p_{7}-p_{6}=\frac{1}{36}$, so the expected number of steps of gain of horse 7 over horse 6 after $n$ throws of the two dice would be $\frac{n}{36}$, which will grow as $n$ grows. We can see that horse 7's advantage increases considerably for longer tracks (Table 1, Fig. 1). 


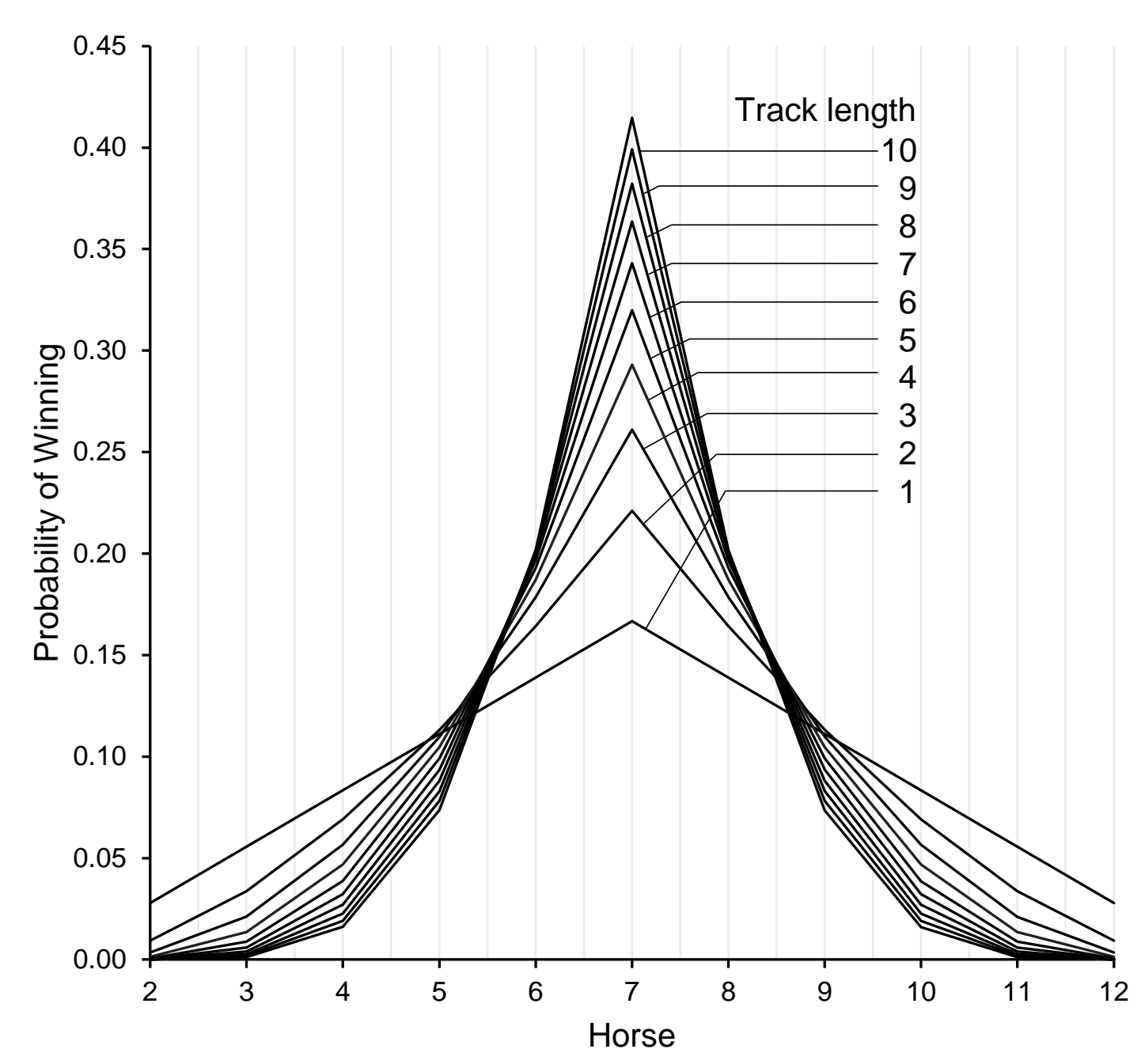

Fig. 1. The probability of each horse winning races of different track lengths.

For the track length of 10 used in the lesson, the probability that horse 7 will win is about 0.42 , which is much greater than $\frac{1}{6}$.

\section{CONCLUSION}

The explanation for horse 7's winning performance in terms of combinations of numbers that sum to 7 is only a small part of the story; the length of the track is also important. From a pedagogical point of view, although using this task to introduce sample space diagrams might be helpful, great care in follow-up questions is needed. To use the two-dice horse race to 10 spaces merely to illustrate the results of a single throw of two dice may be unhelpful. Simplesounding dice scenarios can be much more complicated than they might at first sight appear. 


\section{Acknowledgement}

We would like to thank the editor and the anonymous reviewers for their very helpful comments, as well as Philip O'Neill for helping us with sketching out the details of the Markov analysis in Appendix 2.

\section{Notes}

1. An interactive applet for doing this (with a track length of 12) is available at http://map.mathshell.org/lesson_support/probability_games/index.html. An associated lesson plan is available at http://map.mathshell.org.uk/materials/lessons.php?taskid=596.

2. If the number of horses (not counting horse 1) is $h$ and the track length is $l$, then the largest number of steps to win happens when all horses have made $l-1$ steps, and the winning horse has made one additional step. Hence, we have $h(l-1)+1$ goes, which, for an 11-horse race of track length 2 , is 12 .

3. An Excel macro to perform this simulation is available at http://www.foster77.co.uk/Horse\%20race\%20simulation.xlsm The file is not protected and has extensive comments in the macro to enable students and teachers to develop it further if they wish.

\section{Reference}

Foster, C. (2012). A straight question. Mathematics in School, 41(4), 31-34.

\section{Appendix 1}

We could represent the probability of the $i$ th horse moving one space forward each time by $p_{i}$ (which take the values given above) and then consider the expansion of $\left(\sum_{i=2}^{12} p_{i}\right)^{12}$. Then, to find the probability that the $j$ th horse will win the race, we need to sum terms in the expansion in which $p_{j}$ is raised to a higher power than any other $p_{i}$ and in which the index of $p_{j} \geq 2$. However, the order is important. Imagine a 3-horse race on a track length of 2 , which will require a maximum of 4 rounds for one of the horses to win. The expansion of $\left(p_{1}+p_{2}+\right.$ $\left.p_{3}\right)^{4}$ has terms $p_{1}^{2} p_{2}^{2}$, for instance, but the order $p_{1} p_{1} p_{2} p_{2}$, say, is a win for horse 1 , while $p_{2} p_{2} p_{1} p_{1}$ is a win for horse 2 . So we would need to add in some of these $p_{1}^{2} p_{2}^{2}$ terms but not others, which introduces a further complexity to resolve. In general, using combinatorics, we can say that the probability of horse $j$ winning our race with a track length of 10 on the $n^{\text {th }}$ throw of the dice will be given by:

$\mathrm{P}$ (horse $j$ has moved 9 spaces at round $n-1$; all other horses have moved fewer than 10 spaces at round $n-1) . \mathrm{P}($ horse $j$ moves at round $n$ ).

The first of these probabilities is a constrained sum over the other horses, with multinomial coefficients to count the sequences which are the same, so it is not very easy to calculate! 


\section{Appendix 2}

The full procedure is a vector Markov process. Let $X_{n}=\left(x_{2}, x_{3}, \ldots, x_{12}\right)$, where $n$ is the number of goes so far (i.e., the number of times the dice have been rolled) and $x_{k}$ is the space that the $k$ th horse is on after those $n$ rolls. We start with all horses on the starting line, so $X_{0}=(0,0,0$, $\ldots, 0)$. When we roll the dice, one of the numbers in the vector will increase by 1 . For example, if the first roll gives a total of 4 then we have $X_{1}=(0,0,1,0,0, \ldots, 0)$, because horse number 4 (confusingly, the third horse) moves forward one space while all the others remain where they are. Proceeding in this way gives us a sequence of vectors $X_{1}, X_{2}, X_{3}, \ldots$, which is the vector-valued Markov chain. Now, the probability that the $j$ th number in our vector increases by one at each step is the probability of rolling a total of $j$, which we are calling $p_{j}$, and this gives us the transition probabilities in the Markov chain; i.e., how likely it is to move from any particular state to any other. In this case almost all of the transition probabilities between two states are zero, since from one state there are only ever 11 other states that can be reached, corresponding to the 11 possible dice throws. The race finishes as soon as one of the numbers in the vector reaches 10 , since at that point one of the horses has reached the final square. The standard theory of Markov chains enables us to work out the probability of the chain ending up in a particular state (i.e., with one of the $x_{j}$ s equal to 10 and all the rest less than 10). However, the calculations look prohibitive, because the state space is enormous (around $10^{11}$ ). If the 1-step transition matrix is $\mathrm{P}$, then $\mathrm{P}^{n}$ gives the $n$-step transition matrix, and, since the maximum possible number of rounds is 100 , it follows that $\mathrm{P}^{100}$ gives the probabilities needed.

(Note that it is necessary to define carefully what happens when one horse reaches square 10. The easiest thing is to say that the process then remains where it is from then onwards, to make sure that everything works when you get to $n=100$.) 